Boletín de la Sociedad Geológica Mexicana

VOLUMEN 62, NÚM. 3, 2010, P. 391-398

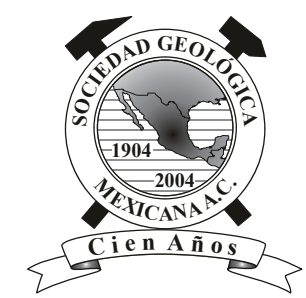

\title{
Variabilidad climática y la acumulación de carbono orgánico en el sector sur de la Corriente de California durante las etapas isotópicas marinas 1,2 y 3
}

\author{
Alberto Sánchez ${ }^{1, *}$, José D. Carriquiry ${ }^{2}$, B. Estela López-Ortiz ${ }^{1}$ \\ ${ }^{1}$ Centro Interdisciplinario de Ciencias Marinas, Departamento de Oceanología, Instituto Politécnico Nacional, La Paz, Baja California \\ Sur, México. \\ ${ }^{2}$ Instituto de Investigaciones Oceanológicas, Universidad Autónoma de Baja California, Ensenada, Baja California, México. \\ *alsanchezg@ipn.mx; alsanchezg13@gmail.com
}

\section{Resumen}

La bomba biológica en el océano desempeña un papel muy importante en el ciclo de carbono global. Las tasas de enterramiento de carbono orgánico $\left(\mathrm{C}_{\mathrm{org}}\right)$ al sur de los $40^{\circ}$ de latitud norte en el Pacífico nororiental sugieren una mejor preservación de la productividad exportada durante el último periodo glaciar. La compilación de datos sobre la tasa de acumulación de $\mathrm{C}_{\text {org }}$ permitió extender el registro del Pacífico nororiental al sector sur de la Corriente de California, que corresponde al Pacífico mexicano durante las etapas isotópicas marinas 1, 2 y 3 (EIM-1, 2 y 3). Los resultados de la tasa de acumulación del $\mathrm{C}_{\text {org }}$ mostraron un incremento en la productividad exportada durante la EIM-2, excepto para el margen de Mazatlán. Estas evidencias sugieren una mejor utilización de los nutrimentos a latitudes medias durante la EIM-2. El aumento en la disponibilidad de nutrientes a la zona fótica, que favoreció la productividad marina y exportada, fue ocasionado por un incremento en las condiciones de surgencia.

Palabras clave: Corriente de California, carbono orgánico, bomba biológica, productividad exportada, etapas isotópicas marinas.

\begin{abstract}
The biological pump of the ocean plays a very important role in the global carbon cycle. The burial rates of organic carbon $\left(C_{\text {org }}\right)$ south of latitude $40^{\circ} \mathrm{N}$ in the eastern North Pacific suggest a better preservation of export productivity during the last glacial cycle. The data compiled for the $C_{\text {org }}$ burial rate allowed us to extend the record of marine isotope stages 1, 2 and 3 (MIS 1,2,3) in the eastern North Pacific to the southern sector of the California Current, which corresponds to the Mexican Pacific. The results of the burial rates of $C_{\text {org }}$ show an increase in the export productivity during the MIS-2, excepting for the Mazatlan margin. This evidence suggests there is better nutrient utilization at mid-latitudes during the MIS-2. The increase of nutrient availability to the photic zone, which favored marine and export productivity, was caused by an increase in upwelling intensity.
\end{abstract}

Keywords: California current, organic carbon, biological pump, export productivity, marine isotope stages. 


\section{Introducción}

Las condiciones de alta productividad y el balance de las masas de agua del Pacífico Norte y ecuatorial han sido ampliamente utilizadas para explicar las variaciones en la intensidad de la zona de oxígeno mínimo $\left(\mathrm{ZOM}, \mathrm{O}_{2}\right.$ $<20 \mu \mathrm{M}$, a $1 \mu \mathrm{M}$ en el núcleo) en el Pacífico nororiental en escalas de tiempo glaciar-interglaciar. Las primeras evidencias de la fluctuación de la ZOM provienen del índice de bioturbación en Cuenca Santa Bárbara, California (Behl, 1995), donde la secuencia sedimentaria consiste en intercalaciones de sedimentos bioturbados y laminados que permitieron inferir los cambios en el nivel de oxigenación de la columna de agua durante el último periodo glaciar (Behl y Kenneth, 1996).

Los registros sedimentarios colectados a lo largo del Pacífico nororiental (PNO), desde Vancouver hasta el margen de California, han mostrado diferencias en el enterramiento de carbono orgánico $\left(\mathrm{C}_{\mathrm{org}}\right)$, que sugieren baja productividad primaria y exportada en el último periodo glaciar (Lyle et al., 1992; Sancetta et al., 1992; Dean et al., 1997; Gardner et al., 1997; Ortiz et al., 1997). No obstante, otros estudios del PNO indicaron que este periodo reveló una alta productividad exportada entre California y el margen mexicano (Pedersen, 1983; Lyle et al., 1988; Gardner et al., 1997; Mortyn y Thunell, 1997; Hendy et al., 2004; Kienast et al., 2004; Sánchez y Carriquiry, 2007a, b). De hecho, la desglaciación estuvo caracterizada por un incremento significativo en el enterramiento de $\mathrm{C}_{\text {org }}$ en los sitios cercanos a la formación de agua intermedia del Pacfico Norte, al mismo tiempo que se depositaron sedimentos laminados en el PNO (e.g., Crusius et al., 2004). Esto sugiere una mejor preservación de $\mathrm{C}_{\mathrm{org}}$ en sedimentos a lo largo del margen nororiental del Pacífico Norte. Si esta hipótesis es verdadera, los registros sedimentarios a lo largo del Pacífico mexicano deberán mostrar cambios en la tasa de enterramiento de $\mathrm{C}_{\text {org }}$ para las etapas isotópicas marinas 1,2 y 3 (EIM-1, 2 y 3).

Ortiz et al. (2004) sugieren que en el periodo de 50 a $11 \mathrm{ka}$, los cambios en la productividad marina ocurridos en el margen de Magdalena se caracterizaron por condiciones oceanográficas similares a las que ocurren durante los eventos de El Niño-La Niña, con una nutriclina somera y productividad elevada durante episodios cálidos y lo contrario en periodos fríos (similar a las condiciones de productividad ocurridas durante El Niño). Sin embargo, Stott et al. (2000) propusieron que la secuencia de sedimentos laminados a lo largo del PNO puede ser interrumpida por cambios en el flujo de $\mathrm{C}_{\text {org }}$ al sedimento, derivados de un decremento en la productividad primaria y exportada, sin necesidad de un cambio en la oxigenación (i.e., ventilación) de la columna de agua a profundidad intermedia, como lo indicaron Behl y Kenneth (1996). De hecho, la comparación de Mo y $\mathrm{Cd}$ sugiere que la productividad puede variar hasta en un factor de 2 fuera de Baja California en los últimos 52 ka (Dean et al., 2006).
Schmittner et al. (2007) sugirieron que la variación de la ZOM en el océano PNO puede ser explicada por un cambio en el acarreo de nutrientes hacia el océano Pacífico, lo que pudo llevar a una disminución en la productividad primaria y exportada y a un descenso en la tasa de respiración de la materia orgánica a lo largo de la columna de agua.

En el presente trabajo se realizó la compilación de los datos de la acumulación de carbono orgánico de una serie de 5 núcleos de sedimento recolectados en el Pacífico subtropical mexicano, entre la zona fronteriza de México con Estados Unidos y el margen de Mazatlán. El objetivo de esta compilación es describir y comparar cómo la tasa de enterramiento de $\mathrm{C}_{\text {org }}$ varió con la latitud y la profundidad de colecta a lo largo del margen subtropical mexicano durante las EIM-1, 2 y 3.

\section{Sitios de colecta}

El Pacífico mexicano está caracterizado por una ZOM bien definida entre los 300 y $800 \mathrm{~m}$ de profundidad. La concentración de oxígeno se incrementa rápidamente por encima de los $300 \mathrm{~m}$ y por debajo de los $800 \mathrm{~m}$ de profundidad, producto de la intensa mezcla en la capa superficial del océano y el arribo de masas de agua enriquecidas en oxígeno que ventilan la columna de agua, respectivamente. Asimismo, la concentración de oxígeno aumenta con el cambio de latitud, siendo más evidente hacia la región polar y en menor medida hacia la ecuatorial. La asimetría en la distribución del oxígeno con la latitud y la profundidad es producto de la formación de agua intermedia del Pacífico Norte y de la baja concentración de oxígeno del agua subsuperficial subtropical que en su origen tiene alta concentración de oxígeno, el cual es consumido por la oxidación de la materia orgánica aportada por la productividad oceánica conforme viaja hacia el margen continental mexicano (e.g., van Geen et al., 2003).

Los núcleos sedimentarios utilizados en el presente estudio fueron colectados a lo largo del Pacífico mexicano en diferentes profundidades y años (Figura 1). Las características específicas de cada uno de los núcleos se presentan en la Tabla 1. Cada uno de ellos fue fechado radiométricamente con ${ }^{14} \mathrm{C}$, lo que permitió establecer los límites de las EIM-1, 2 y 3 con excepción del núcleo Y74-222 cuyos límites de edad fueron establecidos por correlación con el modelo de edad del núcleo LEG 167 ODP-SITE 1020 (para detalles, ver Lyle et al., 2000). Los sitios de colecta estuvieron caracterizados por concentraciones de oxígeno disuelto menores de $5 \mu \mathrm{mol} / \mathrm{kg}$ para los núcleos GC31/PC08, NH15P y NH8P, mientras que los de colecta ODP-Site 1011, NH22P y Y71-2-22 presentaban una concentración de oxígeno de 75, 100 y $125 \mu \mathrm{mol} / \mathrm{kg}$, respectivamente.

La tasa de sedimentación fue muy variable para cada núcleo (Tabla 1); pero fue mayor en el sitio somero del margen de Magdalena con $30 \mathrm{~cm} / \mathrm{ka}$ (van Geen et al., 


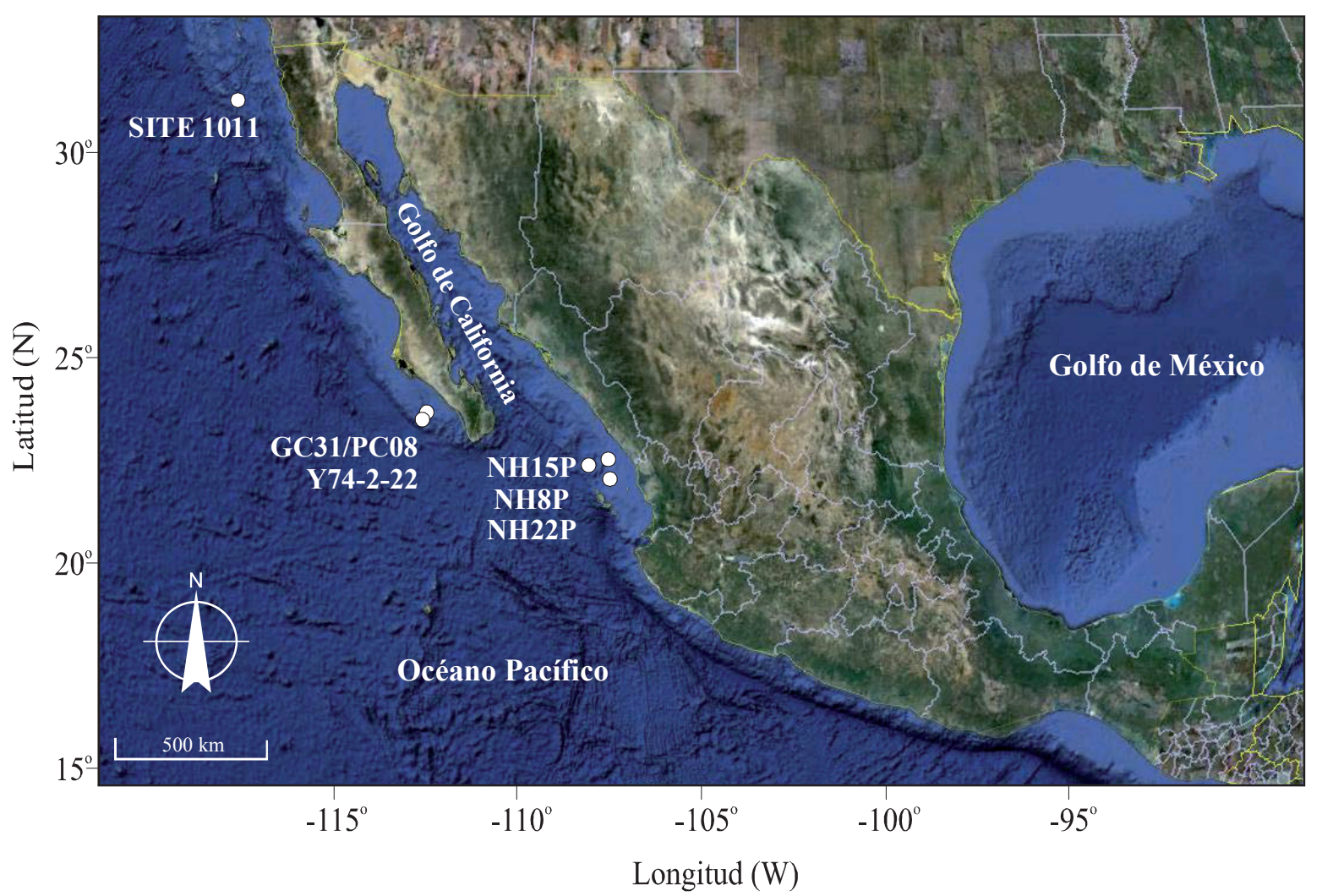

Figura 1. Localización de los sitios de colecta de los núcleos ODP167-SITE 1011 (Lyle et al., 2000); GC31/PC08 (Sánchez y Carriquiry, 2007a, b); Y742-22 (Lyle et al., 2000) y NH15P, 22P y 8P (Ganeshram y Pedersen, 1998) a lo largo del Pacífico oriental mexicano.

Tabla 1. Sitios de colecta de los núcleos sedimentarios con su posición geográfica, profundidad de colecta, concentración de oxígeno disuelto y tasa de sedimentación a lo largo del Pacífico oriental mexicano.

\begin{tabular}{lcccccc}
\hline \multicolumn{1}{c}{ Sitio } & Núcleo & $\begin{array}{c}\text { Latitud } \\
\left({ }^{\circ} \mathbf{N}\right)\end{array}$ & $\begin{array}{c}\text { Longitud } \\
\left({ }^{\circ} \mathbf{W}\right)\end{array}$ & $\begin{array}{c}\text { Profundidad } \\
(\mathbf{m})\end{array}$ & $\begin{array}{c}{\left[\mathbf{O}_{2}\right]} \\
(\mu \mathbf{m o l} / \mathbf{k g})\end{array}$ & $\begin{array}{c}\text { Tasa de } \\
\text { sedimentación } \\
(\mathbf{c m} / \mathbf{k a})\end{array}$ \\
\hline Cuenca Animal & SITE 1011 & $31^{\circ} 16.82^{\prime}$ & $117^{\circ} 38.01^{\prime}$ & 2022 & 75 & 6.5 \\
Margen de Magdalena & $\mathrm{GC}-31 / \mathrm{PC} 08$ & $23^{\circ} 28.20^{\prime}$ & $112^{\circ} 36.00^{\prime}$ & 686 & 2.3 & 30 \\
Margen de Magdalena & $\mathrm{Y} 74-2-22$ & $23^{\circ} 43.80^{\prime}$ & $112^{\circ} 26.28^{\prime}$ & 3054 & 125 & 2.5 \\
Margen de Mazatlán & $\mathrm{NH} 15 \mathrm{P}$ & $22^{\circ} 4.00^{\prime}$ & $106^{\circ} 28.80^{\prime}$ & 420 & $<5$ & 8 \\
Margen de Mazatlán & $\mathrm{NH} 8 \mathrm{P}$ & $22^{\circ} 23.11^{\prime}$ & $107^{\circ} 04.18^{\prime}$ & 1018 & 17 & 15 \\
Margen de Mazatlán & $\mathrm{NH} 22 \mathrm{P}$ & $23^{\circ} 31.04^{\prime}$ & $106^{\circ} 31.04^{\prime}$ & 2025 & 100 & 6 \\
\hline
\end{tabular}

2003). En los sitios de colecta más profundos la tasa de sedimentación fue menor, como lo muestran los núcleos de cuenca Animal, margen de Mazatlán y Magdalena.

\section{Marco analítico}

La determinación de $\mathrm{C}_{\text {org }}$ en los núcleos ODP-SITE1011 de cuenca Animal y Y74-2-22 del margen de Magdalena (Lyle et al., 2000) fue realizada en un analizador elemental Carlo Erba, mientras que los datos de $\mathrm{C}_{\text {org }}$ del núcleo GC31/ PC08 (Sánchez y Carriquiry, 2007a, b) fueron determinados utilizando un analizador elemental LECO. La concentración de $\mathrm{C}_{\text {org }}$ en los núcleos NH15P, NH8P y NH22P en el margen de Mazatlán (Ganeshram y Pedersen, 1998) fue determinada con un analizador elemental Carlo Erba. 


\section{Tasa de acumulación}

La tasa de acumulación de $\mathrm{C}_{\text {org }}$ de cada núcleo fue calculada a partir de la densidad del sedimento seco (DSS $=$ $1.563 \times \mathrm{DSH}-1.560$, donde DSH es densidad del sedimento húmedo; Lyle et al. 2000). La tasa de sedimentación y el porcentaje en peso de los componentes biogénicos fueron multiplicados por DSS para estimar la tasa de acumulación de $\mathrm{C}_{\text {org }}\left(\mathrm{mg} \mathrm{cm}^{-2} \mathrm{ka}^{-1}\right)$.

\section{Registro sedimentario marino}

\subsection{Cuenca Animal - ODP-SITE 1011}

Los valores de $\mathrm{C}_{\text {org }}$ para este núcleo estuvieron en un intervalo de 50 a $90 \mathrm{mg} \mathrm{cm}^{-2} \mathrm{ka}^{-1}$ (Figura 2). La acumulación de $\mathrm{C}_{\text {org }}$ fue muy similar en las EIM-1 y 2; en la EIM-3 la tasa de acumulación de $\mathrm{C}_{\text {org }}$ decreció 20\% (Tabla 2).

\subsection{Margen de Magdalena - GC31/PC08 y Y74-2-22}

La tasa de acumulación de $\mathrm{C}_{\text {org }}$ entre ambos núcleos es significativamente diferente en magnitud con respecto de la profundidad de muestreo (Figura 2; Tabla 2). Por ejemplo, la tasa de acumulación de $\mathrm{C}_{\text {org }}$ en el núcleo GC31/PC08 varió entre 500 y $1450 \mathrm{mg} \mathrm{cm}^{-2} \mathrm{ka}^{-1}$, mientras que en el núcleo Y74-2-22 la tasa de acumulación de $\mathrm{C}_{\text {org }}$ osciló entre $16 \mathrm{y}$ $40 \mathrm{mg} \mathrm{cm}^{-2} \mathrm{ka}^{-1}$ (Figura 2). En las EIM-1 y 2, las tasas de enterramiento de $\mathrm{C}_{\text {org }}$ fueron muy similares para cada sitio. En la EIM-3, la tasa de acumulación tiende a ser menor en el núcleo Y74-2-22 respecto de las otras etapas; mientras que en el núcleo GC31/PC08 fue sólo ligeramente mayor que el resto de las etapas. Específicamente, la tasa de sepultamiento de $\mathrm{C}_{\text {org }}$ fue muy semejante entre el Holoceno y el EIM-2 a

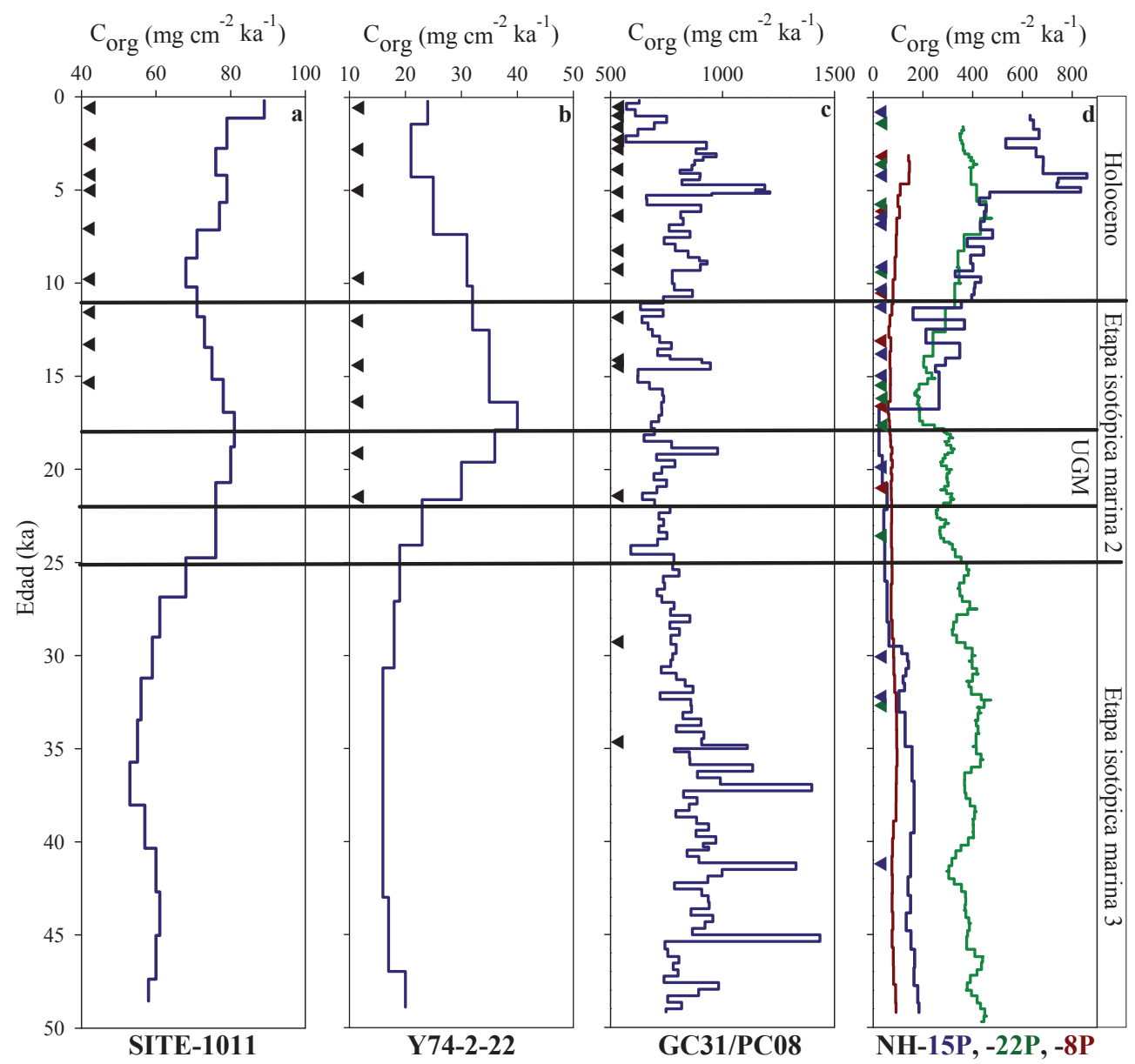

Figura 2. Tasa de acumulación ( $\mathrm{mg} \mathrm{cm}^{-2} \mathrm{ka}^{-1}$ ) del carbono orgánico para los núcleos: (a) ODP167-SITE 1011 (Lyle et al., 2000); (b) Y74-2-22 (Lyle et al., 2000); (c) GC31/PC08 (Sánchez y Carriquiry, 2007a, b) y (d) NH15P, 22P y 8P (Ganeshram y Pedersen, 1998) a lo largo del Pacífico oriental mexicano. Los triángulos negros cercanos al eje de las ordenadas representan los fechados de ${ }^{14} \mathrm{C}$ para los núcleos SITE 1011, Y74-2-22, y GC31/PC08; los azules para el núcleo NH15P; los verdes para el NH22P y los rojos para el NH8P. Los fechados de ${ }^{14} \mathrm{C}$ fueron realizados en foraminíferos planctónicos y bentónicos. UGM: Ultimo Glaciar Máximo. 
Tabla 2. Tasa de acumulación de carbono orgánico $\left(\mathrm{mg} \mathrm{cm}^{-2} \mathrm{ka}^{-1}\right)$ promedio $(\xi)$ y desviación estándar $(\sigma)$ para los diferentes sitios de colecta y etapas isotópicas marinas (EIM-1, 2 y 3).

\begin{tabular}{ccccccccccccc}
\hline & Cuenca Animal & \multicolumn{4}{c}{ Margen Magdalena } & \multicolumn{4}{c}{ Margen Mazatlán } \\
Periodo & SITE 1011 & \multicolumn{1}{c}{ GC31/PC08 } & Y-74-2-22 & \multicolumn{2}{c}{ NH15 P } & \multicolumn{2}{c}{ NH8P } & \multicolumn{2}{c}{ NH22P } \\
\hline & $\xi$ & $\sigma$ & $\xi$ & $\sigma$ & $\xi$ & $\sigma$ & $\xi$ & $\sigma$ & $\xi$ & $\sigma$ & $\xi$ & $\sigma$ \\
EIM-1 & 74 & 5 & 791 & 139 & 25 & 4 & 537 & 156 & 378 & 34 & 107 & 25 \\
EIM-2 & 76 & 4 & 705 & 84 & 33 & 5 & 189 & 130 & 300 & 16 & 70 & 5 \\
EIM-3 & 59 & 4 & 872 & 141 & 17 & 2 & 136 & 33 & 386 & 38 & 84 & 8 \\
\hline
\end{tabular}

lo largo de la península de Baja California.

\subsection{Margen de Mazatlán - NH15P, NH8P y NH22P}

Las tasas de acumulación de $\mathrm{C}_{\text {org }}$ fueron muy variables entre núcleos y para una misma EIM (Figura 2, Tabla 2). Los núcleos NH15P y NH22P mostraron una mayor tasa de acumulación de $\mathrm{C}_{\text {org }}$ durante la EIM-1 que en el resto de las EIM, mientras que el núcleo NH8P tuvo un valor más alto en la EIM-3. La tasa de acumulación disminuye conforme aumenta la profundidad para los núcleos NH22P y NH8P. Particularmente, la tasa de acumulación del núcleo NH15P fue mayor en la EIM-1 que la de los núcleos NH8P y NH22P, pero menor que el núcleo NH8P en la EIM-2 y 3 (Tabla 2).

\section{Discusión}

La acumulación de carbono orgánico, así como de otros trazadores geoquímicos, ha sido utilizada para clarificar la historia de la producción exportada en el Pacífico Norte. Cada herramienta biogeoquímica proporciona información valiosa sobre el ciclo del carbono, sin embargo tienen particularidades y problemas por lo que deben ser usadas con cautela. Numerosos estudios han demostrado una excelente relación positiva entre la productividad primaria, la exportación y el enterramiento de $\mathrm{C}_{\text {org }}$ (e.g., Müller y Suess, 1979; Sarnthein et al., 1987; Calvert et al., 1992, 1995; Seiter et al., 2004; Sánchez y Carriquiry, 2007a, b; Paytan, 2008), no obstante las condiciones exactas bajo las cuales el $\mathrm{C}_{\text {org }}$ es preservado en el sedimento son objeto de debate (Hartnett et al., 1998; Stott et al., 2000; Thunell et al., 2000; Hedges et al., 2001; Seiter et al., 2004). La tasa de sedimentación alta (e.g., Müller y Suess, 1979), así como la protección física de la materia orgánica por una matriz inorgánica de partículas que se hunden (Hedges et al., 2001), son hipótesis que se han planteado para explicar la preservación de $\mathrm{C}_{\text {org }}$; mientras que la exposición al oxígeno (Hartnett et al., 1998) se ha propuesto para explicar la disminución de $\mathrm{C}_{\text {org }}$ (por problemas de preservación). Recientemente, se ha demostrado que la tasa a la cual la materia orgánica lábil es degradada en la columna de agua es la misma tanto para cuencas anóxicas como para el océano abierto (Thunell et al., 2000), indicando que el tiempo de exposición al oxígeno no es el factor dominante sobre la preservación de la materia orgánica sedimentaria. Asimismo, en un estudio realizado en algunas cuencas del PNO, Stott et al. (2000) resaltaron la importancia de la productividad superficial como control, no sólo del contenido de $\mathrm{C}_{\text {org }}$ en sedimento, sino también del contenido de oxígeno en el agua de fondo. Estos autores mostraron que la superposición de sedimentos bioturbados sobre sedimentos laminados fue causada por una reducción total en las surgencias costeras (i.e., reducción de producción marina) durante el transcurso del siglo XX. Lyle et al. (1992) compararon la tasa de acumulación de $\mathrm{C}_{\text {org }}$ en trampas de sedimento localizadas a $1000 \mathrm{~m}$ de profundidad con la del margen de Oregon, E.U.A., y encontraron que sólo $20 \%$ del flujo de $\mathrm{C}_{\text {org }}$ total es preservado en el sedimento superficial.

Adicionalmente, el hundimiento de $\mathrm{C}_{\text {org }}$ es controlado por el arrastre de partículas asociadas y no asociadas a minerales (Oka et al., 2008). Thunell et al. (2007) evaluaron la ballast hypothesis para una serie de trampas de sedimento en las cuencas de Santa Bárbara, Guaymas y Cariaco. Los flujos de $\mathrm{C}_{\text {org }}$ están fuertemente correlacionados con los minerales (carbonato, ópalo y litogénicos) en los tres sitios. Esto apoya la ballast hypothesis, donde se ha sugerido que los minerales de mayor densidad optimizan el flujo de materia orgánica al fondo del océano (Klaas y Archer, 2002). Con base en un análisis de regresión múltiple, los tres componentes minerales explican el $72 \%$ de la varianza total en el flujo de $\mathrm{C}_{\text {org }}$ en los tres sitios, donde las partículas de carbonato biogénico parecen ser las más eficaces para el transporte, tanto en el océano abierto (Klaas y Archer, 2002) como a lo largo de los márgenes continentales (MullerKarger et al., 2005; Thunell et al., 2007).

La tasa de acumulación de $\mathrm{C}_{\text {org }}$ a lo largo del Pacífico oriental mexicano (POM) mostró una tendencia a disminuir conforme incrementa la profundidad, lo que es evidente para los márgenes de Magdalena y Mazatlán durante la EIM-1. Este mismo comportamiento se observa para el margen de Magdalena en las EIM-2 y 3, pero no así para el margen de Mazatlán, donde la tasa de acumulación de $\mathrm{C}_{\text {org }}$ se mantiene sin variación en el núcleo NH8P a lo largo de las EIM-1, 2 y 3. Estudios previos describen la distribución de $\mathrm{C}_{\text {org }}$ en sedimentos marinos y los mapas generalizados refieren claramente que los márgenes continentales están caracterizados por altos contenidos de $\mathrm{C}_{\text {org }}$ que decrecen con la distancia y profundidad en la mayoría de las cuencas oceánicas (Seiter et al., 2004). 
La fluctuación observada en la tasa de enterramiento de $\mathrm{C}_{\text {org }}$ ha originado dos posibles hipótesis para explicar como la bomba biológica - transferencia de $\mathrm{C}_{\text {org }}$ al océano profundo por hundimiento del detritus biogénico - influyó en la concentración de $\mathrm{CO}_{2}$ atmosférico. (e.g., Beaufort et al., 2001). La primera es sustentada por estudios que sugieren que la bomba biológica en latitudes bajas fue más eficiente durante la EIM-2 debido a que el reservorio de nutrientes no fue limitante (e.g., Paytan et al., 1996). En la segunda hipótesis varios autores proponen que la bomba biológica fue más eficaz durante los periodos glaciares porque los nutrientes se utilizaron completamente en latitudes medias y altas, mientras que muchos permanecieron inutilizados durante periodos interglaciares (e.g., Kienast et al., 2004).

Kienast et al. (2004) recopilaron una serie de núcleos del Pacífico Norte, dos de los cuales fueron colectados en la región norte del giro subtropical mostrando evidencias de un incremento en la productividad exportada durante el periodo glacial. Esto contrastó con los datos de la región subpolar que revelaron baja productividad exportada. Estos autores indican que no hay registros en el Pacífico subtropical sobre cambios en la productividad exportada que permitan extender estos registros hacia las regiones centrales de los giros subtropicales. No obstante, algunos registros a lo largo del margen occidental de Norteamérica (Oregon, California, Baja California y Sinaloa) han reportado que la productividad exportada tuvo un cambio latitudinal como el observado por Kienast et al. (2004). Al norte de los $40^{\circ}$ de latitud norte la productividad exportada fue menor durante la EIM-2 (Lyle et al., 1992; Sancetta et al., 1992; Dean et al., 1997; Gardner et al., 1997; Ortiz et al., 1997) mientras que al sur de $\operatorname{los} 40^{\circ}$ fue significativamente mayor en la EIM2 que en el Holoceno (Pedersen, 1983; Lyle et al., 1988; Gardner et al., 1997; Mortyn y Thunell, 1997; Hendy et al., 2004; Kienast et al., 2004; Sánchez y Carriquiry, 2007a, b; Carriquiry y Sánchez, 2010).

En el caso particular del POM, la tasa de acumulación de $\mathrm{C}_{\text {org }}$ durante la EIM-2 fue similar o ligeramente mayor a la obtenida en la EIM-1, con excepción de los núcleos NH15P y NH22P, y ligeramente menor para el núcleo NH8P. Específicamente, los registros sedimentarios a lo largo de Baja California, bajo la influencia de la Corriente de California, mostraron un incremento en la productividad exportada durante la EIM-2. Kienast et al. (2004) sugirieron que la productividad exportada incrementó al sur de los $40^{\circ}$ de latitud, sin embargo su proposición es limitada debido a que no incluyeron otros registros, como los núcleos colectados a lo largo del margen de California, donde recientemente (Sánchez y Carriquiry, 2007a, b; Carriquiry y Sánchez, 2010) se encontró un incremento en la productividad exportada para el margen de Magdalena, Baja California Sur. Los datos compilados en el presente trabajo confirman que el incremento de la productividad exportada puede extenderse desde los $40^{\circ}$ hasta los $20^{\circ}$ de latitud norte, con excepción de dos de los tres núcleos, particularmente frente a Mazatlán.
Los resultados de la acumulación de $\mathrm{C}_{\text {org }}$ en sedimentos del POM apoyan las evidencias propuestas por Kienast et al. (2004), quienes sugieren una utilización completa y eficiente de los nutrientes a latitudes medias durante la EIM-2. No obstante, la disponibilidad de nutrientes para la zona fótica depende de ciertos mecanismos oceanográficos (e.g., surgencias), a partir de los cuales es posible un incremento en la productividad marina y exportada. La tasa de acumulación de $\mathrm{C}_{\text {org }}$ puede ser una herramienta geoquímica confiable para estimar productividad marina y exportada de épocas pasadas (e.g., Paytan, 2008), sin embargo tiene ciertas limitaciones, por lo que debe ser usada con cautela o ser estimada mediante el uso simultáneo de varios indicadores, y así fortalecer las interpretaciones (e.g., Hedges et al., 2001; Seiter et al., 2004).

Particularmente en el núcleo NH8P, fuera del margen de Mazatlán, el flujo de Corg fue de $378 \pm 34 \mathrm{mg} \mathrm{cm}^{-2} \mathrm{ka}^{-1}$ y $296 \pm 25 \mathrm{mg} \mathrm{cm}^{-2} \mathrm{ka}^{-1}$, y el ópalo biogénico fue $300 \pm 16$ $\mathrm{mg} \mathrm{cm}{ }^{-2} \mathrm{ka}^{-1}$ y $243 \pm 24 \mathrm{mg} \mathrm{cm}^{-2} \mathrm{ka}^{-1}$ para el Holoceno y para el EIM-2, respectivamente. Estos flujos no fueron significativamente diferentes entre periodos. De hecho, los porcentajes de ópalo biogénico para el núcleo ODP-SITE 1017 fueron $5.8 \pm 0.4 \%$ y $6.0 \pm 0.4 \%$ durante el Holoceno y EIM2, respectivamente, en el margen de California (Hendy et al., 2004). Sánchez y Carriquiry (2007a) reportaron flujos de ópalo biogénico para la EIM-1 de $1480 \pm 297 \mathrm{mg} \mathrm{cm}^{-2} \mathrm{ka}^{-1}$ y para la EIM2 de $2079 \pm 348 \mathrm{mg} \mathrm{cm}^{-2} \mathrm{ka}^{-1}$ en el margen de Magdalena. Estas evidencias sugieren que los márgenes de California y Baja California experimentaron durante la EIM2 una productividad muy similar a otros estudios mencionados para la EIM-1.

En efecto, el POM está caracterizado por la presencia de una intensa zona de oxígeno mínimo que promueve la pérdida de $\mathrm{N}$ debido a la desnitrificación de la columna de agua, lo que limita la productividad primaria (Ganeshram et al., 2002). Algunos estudios realizados en esta región han demostrado que la pérdida de $\mathrm{N}$ disminuyó durante la EIM-2 (Ganeshram et al., 2002; Thunell y Kepple, 2004) promoviendo un incremento en el reservorio de N, y aparentemente, un aumento de la productividad primaria. Tyrrell (1999) indicó que el fitoplancton requiere de proporciones constantes de $\mathrm{N}$ y $\mathrm{P}$, y sugirió que una razón N/P mayor a la razón de Redfield puede suprimir la fijación de N. Por lo que una razón de N/P baja indicaría la fijación de $\mathrm{N}$ favoreciendo la productividad, debido a que tiende a balancear la pérdida de $\mathrm{N}$ a causa de la desnitrificación (Haug et al., 1998).

La razón N/P en el registro sedimentario del margen de Magdalena fue baja durante la EIM-2, con valores muy cercanos al Holoceno tardío (Figura 5 en Sánchez y Carriquiry, 2007a), lo cual concuerda con lo propuesto por Ganeshram et al. (2002) y Haug et al. (1998) sobre una mejor utilización del $\mathrm{N}$ a bajas razones del N/P. De esta forma, la fijación de $\mathrm{N}$ y el contenido de $\mathrm{P}$ no fueron limitantes durante la EIM-2, llevando a una productividad primaria similar en la margen de Magdalena. De hecho, 
la razón $\mathrm{BSi} / \mathrm{C}$ es máxima y sincrónica con periodos de intensas surgencias y alta productividad primaria, dominada principalmente por diatomeas, y esta razón decrece con una columna de agua estratificada y productividad baja (Goñi et al., 2003). En el margen de Magdalena la razón $\mathrm{BSi} / \mathrm{C}$ fue similar para la EIM2 y el Holoceno tardío (Figura 5 en Sánchez y Carriquiry, 2007a). Estas evidencias sugieren que las condiciones de surgencias fueron tan favorables e intensas durante la EIM-2 como lo fueron en el Holoceno tardío. De manera general, la relación inversa entre las razones $\mathrm{N} / \mathrm{P}$ y $\mathrm{BSi} / \mathrm{C}$ concuerda con lo propuesto sobre una mejor utilización y asimilación de los nutrientes por parte las diatomeas, en el margen de Magdalena durante la EIM-2 y UGM.

Sánchez y Carriquiry (2007a) propusieron un mecanismo oceanográfico que favorece la disponibilidad de nutrientes hacia la fótica durante la EIM-2. Estos autores utilizaron las interpretaciones realizadas de los modelos atmosféricos del grupo de COHMAP (1988) y Kutzbach (1987). Los resultados de estos dos modelos sugirieron que la celda de alta presión del Pacífico norte pudo haberse desplazado hacia una posición más al sur durante la EIM-2, lo que ocasionó una intensificación de los vientos a lo largo del margen continental, entre las latitudes de $40^{\circ}$ y $20^{\circ}$ norte, que favoreció las condiciones de surgencia que permitieron la disponibilidad de nutrimentos, y de esta forma incrementar la productividad marina y exportada durante la EIM-2 (Gardner et al., 1997; Mortyn y Thunell, 1997; Hendy et al., 2004).

\section{Conclusiones}

La tasa de acumulación de Corg disminuye con el incremento de la profundidad en los márgenes de Magdalena y Mazatlán. El incremento en la tasa de acumulación de Corg entre las latitudes de $40^{\circ}$ y $20^{\circ}$ norte, a lo largo del Pacífico nororiental de California y Baja California, sugiere que existió una mejor utilización de los nutrientes durante la EIM-2, lo que apoya la hipótesis propuesta por Kienast et al. (2004). La disponibilidad de nutrientes hacia la zona fótica fue posiblemente ocasionada por surgencias costeras a lo largo del margen continental del Pacífico oriental mexicano generando un incremento en la productividad marina y exportada durante la EIM-2, respecto del Holoceno.

\section{Referencias}

Beaufort, L., de Garidel-Thoron, T., Mix, A.C., Pisias, N.G., 2001, ENSO-like forcing on oceanic primary production during the Late Pleistocene: Science, 293, 2440-2444.

Behl, R.J., 1995, Sedimentary facies and sedimentology of the Late Quaternary Santa Barbara Basin (Site 893): Proceedings of the Ocean Drilling Program Science Results, 146, 295-308.

Behl, R.J., Kennett, J.P., 1996, Brief interstadial events in the Santa Barbara Basin, NE Pacific during the past 60 Kyr: Nature, 379, 243-246.

Calvert, S.E., Bustin, R.M., Pedersen, T.F., 1992, Lack of evidence for enhanced preservation of sedimentary organic matter in the oxygen minimum of the Gulf of California: Geology, 20, 757-760.

Calvert, S.E., Pedersen, T.F., Naidu, P.D., Stackelberg, U., 1995, On the organic carbon maximum on the continental slope of the eastern Arabian Sea: Journal of Marine Research, 53, 269-296.

Carriquiry, J., Sánchez, A., 2010, Ópalo biogénico sedimentario en el extremo Sur del sistema de surgencias de la Corriente de California: etapas isotópicas marinas 1-5, en Gaxiola-Castro, G., DurazoArvizu, R. (eds.), Dinámica del ecosistema pelágico frente a Baja California: México, D.F., Instituto Nacional de Ecología: 227-238.

Cooperative Holocene Mapping Project (COHMAP) members, 1988, Climatic changes of the last 18000 years: Observations and model simulations: Science, 241, 1043-1052.

Crusius, J., Pedersen, T.F., Kienast, S., Keigwin, L., Labeyrie, L., 2004, Influence of northwest Pacific productivity on North Pacific Intermediate Water oxygen concentrations during the BøllingAllerød interval (14.7 - $12.9 \mathrm{ka})$ : Geology, 32, 633-636.

Dean, W.E., Gardner, J.V., Piper, D.Z., 1997, Inorganic geochemical indicators of glacial-interglacial changes in productivity and anoxia on the California continental margin: Geochimica et Cosmochimica Acta, 61, 4507-4518.

Dean, W.E., Zheng, Y., Ortiz, J.D., van Geen, A., 2006, Sediment Cd and Mo accumulation in the oxygen-minimum zone off western Baja California linked to global climate over the past $52 \mathrm{kyr}$ : Paleoceanography, 21, PA4209.

Ganeshram, R.S., Pedersen, T.F., 1998, Glacial-interglacial variability in upwelling and bioproductivity off NW Mexico: Implications for Quaternary paleoclimate: Paleoceanography, 13, 634-645.

Ganeshram, R.S., Pedersen, T.F., Calvert, S.E., François, R., 2002, Reduced nitrogen fixation in the glacial ocean inferred from changes in marine nitrogen and phosphorus inventories: Nature, 415, 156-159.

Gardner, J.V., Dean, W.E., Dartnell, P., 1997, Biogenic sedimentation beneath the California Current system for the past $30 \mathrm{kyr}$ and its paleoceanographic significance: Paleoceanography, 12, 207-225.

Goñi, M.A., Aceves, H.L., Thunell, R.C., Tappa, E., Black, D., Astor, Y., Varela, R., Muller-Karger, F., 2003, Biogenic fluxes in the Cariaco Basin: A combined study of sinking particulates and underlying sediments: Deep-Sea Research, 50, 781-807.

Hartnett, H.E., Keil, R.G., Hedges, J.I., Devol, A.H., 1998, Influence of oxygen exposure time on organic carbon preservation in continental margin sediments: Nature, 391, 572-574.

Haug, G.H., Pedersen, T. F., Sigman, D.M., Calvert, S.E., Nielsen, B.,. Peterson, L.C., 1998, Glacial/interglacial variations in production and nitrogen fixation in the Cariaco Basin during the last $580 \mathrm{kyr}$ : Paleoceanography, 13, 427- 432.

Hedges, J.I., Baldock, J.A., Gélinas, Y., Lee, C., Peterson, M., Wakeham, S.G., 2001, Evidence for non-selective preservation of organic matter in sinking marine particles: Nature, 409, 801-804.

Hendy, I.L., Pedersen, T.F., Kennett, J.P., Tada, R., 2004, Intermittent existence of a southern Californian upwelling cell during submillennial climate change of the last $60 \mathrm{kyr}$ : Paleoceanography, 19, 1-15.

Kienast, S.S., Hendy, I.L., Crusius, J., Pedersen, T.F., Calvert, S.E., 2004, Export production in the subarctic North Pacific over the last 800 kyrs: No evidence for iron fertilization?: Journal of Oceanography, 60, 189-203.

Klaas, C., Archer, D.E., 2002, Association of sinking organic matter with various types of mineral ballast in the deep sea: Implications for the rain ratio: Global Biogeochemical Cycles, 16, 1-14.

Kutzbach, J.E., 1987, Model simulations of the climatic patterns during the deglaciation of North America, en Ruddiman, W.F., Wright, H.E. (eds.), North America and adjacent oceans during the Last Deglaciation: Boulder, Colorado, E. U. A., Geological Society of America, 425-446.

Lyle, M., Murray, D.W., Finney, B.P., Dymond, J., Robbins, J.M., Brooksforce, K., 1988, The record of Late Pleistocene biogenic sedimentation in the eastern tropical Pacific Ocean: Paleoceanography, 3, 39-59. 
Lyle, M., Zahn, R., Prahl, F., Dymond, J., Collier, R., Pisias, N., Suess, E., 1992, Paleoproductivity and carbon burial across the California Current: the multitracers transect, $42^{\circ} \mathrm{N}$ : Paleoceanography, 7 , 251-272.

Lyle, M., Mix, A., Ravelo, C., Andreasen, D., Heusser, L., Olivarez, A., 2000, Millennial-scale $\mathrm{CaCO}_{3}$ and $\mathrm{C}$ (org) events along the northern and central California margins: Stratigraphy and origins: Proccedings of the Ocean Drilling Program Science Results, 167, 163-182.

Mortyn, P.G., Thunell, R.C., 1997, Biogenic sedimentation and surface productivity changes in the Southern California Borderlands during the last glacial-interglacial cycle: Marine Geology, 138, 171-192.

Müller, P.J., Suess, E., 1979, Productivity, sedimentation rate, and sedimentary organic matter in the oceans-I. Organic carbon preservation: Deep-Sea Research Part A Oceanographic Research Papers, 26, 1347-1362.

Muller-Karger, F.E., Varela, R., Thunell, R., Luerssen, R., Hu, C., Walsh, J., 2005, The importance of continental margins in the global carbon cycle: Geophysical Research Letters, 32, 1-4.

Oka, A., Kato, S., Hasumi, H., 2008, Evaluating effect of ballast mineral on deep-ocean nutrient concentration by using an ocean general circulation model: Global Biogeochemical Cycles, 22, GB3004.

Ortiz, J., Mix, A., Hostetler, S., Kashgarian, M., 1997, The California Current of the last glacial maximum: Reconstruction at $42^{\circ} \mathrm{N}$ based on multiple proxies: Paleoceanography, 12, 191-205.

Ortiz J.D., O'Connell, S.B., Del Viscio, J., Dean, W., Carriquiry J.D., Marchitto, T.M., Zheng, Y., van Geen, A., 2004, Enhanced marine productivity off western North America during warm climate intervals of the past $52 \mathrm{ky}$ : Geology, 32, 521-524.

Paytan, A., 2008, Ocean Paleoproductivity, en Gornitz, V. (ed.), Encyclopedia of Paleoclimatology and Ancient Environments: New York, Springer, 644-650.

Paytan, A., Kastner, M., Chavez, F.P., 1996, Glacial to interglacial fluctuations in productivity in the Equatorial Pacific as indicated by marine barite: Science, 274, 1355-1357.

Pedersen, T.F., 1983, Increased productivity in the eastern equatorial Pacific during the last glacial maximum (19000 to $14000 \mathrm{yr} \mathrm{BP})$ : Geology, 11, 16-19.

Sancetta, C., Lyle, M., Heusser, L., Zahn, R., Bradbury, J.P., 1992, Lateglacial to holocene changes in winds, upwelling, and seasonal production of the northern California Current system: Quaternary Research, 38, 359-370.

Sánchez, A., Carriquiry, J., 2007a, Acumulación de $\mathrm{C}_{\text {org }}, \mathrm{N}_{\text {org }}, \mathrm{P}_{\text {org }}$ y BSi en la margen de Magdalena, BCS (México), durante los últimos 26 ka: Ciencias Marinas, 33, 23-35.

Sánchez, A., Carriquiry, J., 2007b, Flujos de carbono orgánico sedimentario a lo largo del margen continental del Pacífico nororiental mexicano durante los últimos 50000 años, en Hernández de la Torre, B, Gaxiola Castro, G. (eds.), Carbono en ecosistemas acuáticos de México:
Ensenada, Secretaría de Medio Ambiente y Recursos NaturalesInstituto Nacional de Ecología-Centro de Investigación Científica y de Educación Superior de Ensenada, 427-436.

Sarnthein, M., Winn K., Zahn, R., 1987, Paleoproductivity of oceanic upwelling and the effect on atmospheric $\mathrm{CO} 2$ and climatic change during deglaciation times, en Berger, W.H., Labeyrie, L.D. (eds.), Abrupt Climatic Change. Evidence and implications: Dordrecht, The Netherlands, D. Reidel Publishing Company, 311-337.

Schmittner, A., Galbraith, E.D., Hostetler, S.W., Pedersen, T.F., Zhang, R., 2007, Large fluctuations of dissolved oxygen in the Indian and Pacific oceans during Dansgaard-Oeschger oscillations caused by variations of North Atlantic Deep Water subduction: Paleoceanography, 22, PA3207.

Seiter, K., Hensen, C., Schröter, J., Zabel, M., 2004, Organic carbon content in surface sediments -Defining regional provinces: Deep-Sea Research Part I: Oceanographic Research Papers, 51, 2001-2026.

Stott, L.D. Berelson, W., Douglas, R., Gorsline, D., 2000, Increased dissolved oxygen in Pacific intermediate waters due to lower rates of carbon oxidation in sediments: Nature, 407, 367-370.

Thunell, R.C., Kepple, A.B., 2004, Glacial-Holocene $\delta^{15} \mathrm{~N}$ record from the Gulf of Tehuantepec, Mexico: Implications for denitrification in the eastern equatorial Pacific and changes in atmospheric $\mathrm{N}_{2} \mathrm{O}$ : Global Biogeochemical Cycles, 18, 1-12.

Thunell, R.C., Varela, R., Llano, M., Collister, J., Muller-Karger, F., Bohrer, R., 2000, Organic carbon fluxes, degradation, and accumulation in an anoxic basin: Sediment trap results from the Cariaco Basin: Limnology and Oceanography, 45, 300-308.

Thunell, R., Benitez-Nelson, C., Varela, R., Astor, Y., Muller-Karger, F., 2007, Particulate organic carbon fluxes along upwelling-dominated continental margins: rates and mechanisms: Global Biogeochemical Cycles, 21, GB1022.

Tyrrell, T., 1999, The relative influences of nitrogen and phosphorus on oceanic primary production: Nature, 400, 525- 531.

van Geen, A., Zheng, Y., Bernhard, J.M., Cannariato, K.G., Carriquiry, J., Dean, W.E., Eakins, B.W., Ortiz, J.D., Pike, J., 2003, On the preservation of laminated sediments along the western margin of North America: Paleoceanography, 18, 1098.

Manuscrito recibido: Septiembre 14, 2009.

Manuscrito corregido recibido: Enero 10, 2010.

Manuscrito aceptado: Marzo 12, 2010. 